\title{
Raman Spectroscopy of Irradiated Normoxic Polymethacrylic Acid Gel Dosimeter
}

\author{
Jihye Bong, Kyuseok Choi, ${ }^{\dagger}$ Soo-Chang Yu, ${ }^{\star}$ Soo-Il Kwon, ${ }^{*}$ Yura Cho, Chaehee Park, and Hyung-wook Park \\ Department of Medical Physics, Kyonggi University, Suwon 443-760, Korea. E-mail: sikwon@kyonggi.ac.kr \\ Center for Research Facilities, ${ }^{\dagger}$ Department of Chemistry ${ }^{\ddagger}$ Kunsan National University, Gunsan 573-701, Korea \\ Received October 20, 2010, Accepted December 14, 2010
}

\begin{abstract}
A quantitative analysis of the decreasing rate of the monomer and increasing rate of the polymerization was made by monitoring radiation level increments using Raman spectroscopy within the therapeutic radiation range for a normoxic polymethacrylic acid gel dosimeter. The gel dosimeter was synthesized by stirring materials such as gelatin, distilled water, methacrylic acid, hydroquinone and tetrakis phosphonium chloride at $50{ }^{\circ} \mathrm{C}$, and the synthesized gel was contained in a $10-\mathrm{mm}$ diameter and $32-\mathrm{mm}$ high vial to conduct measurement. 24 hours after gel synthesis, it was irradiated from 0 Gy to 20 Gy by 2 Gy using a Co-60 radiotherapy unit. With use of the Cryo FE-SEM, structural changes in the $0 \mathrm{~Gy}$ and $10 \mathrm{~Gy}$ gel dosimeters were investigated. The Raman spectra were acquired using 532-nm laser as the excitation source. In accordance with fitting the changes in $\mathrm{C}-\mathrm{COOH}$ stretching $\left(801 \mathrm{~cm}^{-1}\right), \mathrm{C}=\mathrm{C}$ stretching $\left(1639 \mathrm{~cm}^{-1}\right)$ and vinyl $\mathrm{CH}_{2}$ stretching $\left(3114 \mathrm{~cm}^{-1}\right)$ vibrational modes for monomer and $\mathrm{CH}_{2}$ bending vibrational mode $\left(1451 \mathrm{~cm}^{-1}\right)$ for polymer, sensitive parameter $\mathrm{S}$ for each mode was calculated. The values of $\mathrm{S}$ for monomer bands and polymer band were ranged in $6.0 \pm 2.6$ Gy and 7.2 $\pm 2.3 \mathrm{~Gy}$, respectively, which shows a relatively good conformity of the decreasing rate of monomer and the increasing rate of polymerization within the range of error.
\end{abstract}

Key Words: Polymer gel, Dosimeter, Polymethacrylic acid, Raman spectroscopy, Cryo FE-SEM

\section{Introduction}

Polymer gel has gathering interest as 3-dimensional measuring equipment for radiation dosage in radiation treatment. Polymer gel creates free radicals by radiation using the principle in which a polymer is created by the polymerization of a monomer, also in which the degree of polymerization differs according to radiation level, so that it is able to be applied as a dosimeter to measure radiation dose. ${ }^{1-3}$ A Polymer gel dosimeter, which measures radiation dose in the human body, should be tissue equivalent in advance. Moreover, it should guarantee dose-response stability, sensitivity and integrity of the dose distribution. ${ }^{4}$ A series of studies for checking chemical changes along radiation level in the gel dosimeter are actively carried out using magnetic resonance imaging (MRI), Optical CT or X-ray CT. ${ }^{5}$ However, it is difficult to fully understand the chemical structure changes of the gel according to radiation doses.

Raman spectroscopy, which examines the orientation of molecules or structural features along with monitoring vibrational mode changes in molecules in application of scattering light from the interaction of light and materials, is a favorable choice to discover information on condition of substance and chemical reaction. ${ }^{6}$ Raman spectroscopy, which is a noninvasive method, is not affected by water unlike infrared (IR) parted in the same vibration spectroscopy, and is also greatly advantageous in the analysis of water soluble substances or biochemical substances owing to its convenience of use, as a pre-processing stage is not necessary. Owing to these advantages it has been applied in studies of gel dosimeters, where monomers are used for acrylamide (AA) and bis-acrylamide (bis-AA), which allows studies about the interaction between monomer consumption of gel dosimeter and polymer. ${ }^{7-9}$ Owing to its lower toxicity as a carcinogen in methacrylic acid (MAA) than AA, interest in gel dosimeters using this substance is increasing. ${ }^{4}$ The study aims to synthesize a polymer gel and indentify radiation action mechanism of gel dosimeter in accordance with analysis of characteristic vibrational mode of MAA monomers through a Raman experiment, which will be applied as basic necessary data to secure a greatly enhanced polymer gel dosimeter.

\section{Experimental}

Gel Dosimeter. The synthetic process for the normoxic polymer gel applied in the study is as follows: $6 \%$ of gelatin (300 bloom, Sigma- Aldrich, USA) is made in distilled water with high purity (HPLC grade water). When the gelatin is fully inflated by the water, a flask is heated while stirring. Before the gelatin is fully dissolved, $8 \%$ of methacrylic acid (MAA, SigmaAldrich, USA) is added, and then $0.05 \mathrm{mM}$ of hydroquinone (HQ, Sigma-Aldrich, USA) and $10 \mathrm{mM}$ of tetrakis (hydroxymethyl) phosphonium chloride (THPC, Sigma-Aldrich, USA) are added in order. When the temperature of the gelatin solution reaches $50{ }^{\circ} \mathrm{C}$, heating is stopped, and the temperature of the gelatin solution is cooled to $43{ }^{\circ} \mathrm{C}$ by putting the reaction flask into a cistern. The whole process is conducted in normoxic conditions, with external air conditioning, without using a glove box or oxygen purging devices.

The completed polymethacylic acid (PMAA) gel solution is contained in a $10-\mathrm{mm}$ diameter with $32-\mathrm{mm}$ high vial, and a gel dosimeter is produced. The gel solution is filled to the top of the vial to minimize oxidation. It is kept at room temperature for 1 hour, and stored at $5{ }^{\circ} \mathrm{C}$ in a refrigerator for gel solidification.

Irradiation. After 24 hours from synthesis of the gel, a dosimeter gel vials were put on a solid water phantom, and examination of the radiation is conducted using a Co-60 radiotherapy unit (Theratron-780; AECL, Ottawa, Canada). With an opening of $30 \times 30 \mathrm{~cm}^{2}$ for radiation examination band, the maximum 

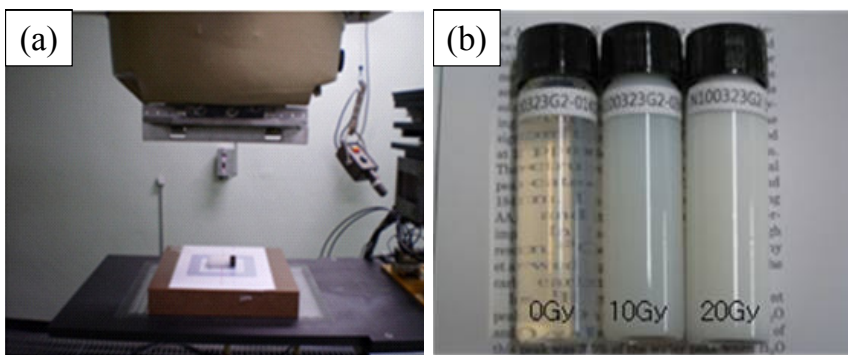

Figure 1. Irradiation of normoxic polymethacrylic acid gels: (a) gels were irradiated by cobalt-60. (b) pre-irradiated samples (left), postirradiated: $10 \mathrm{~Gy}$ (mid) and $20 \mathrm{~Gy}$ (right). (a)

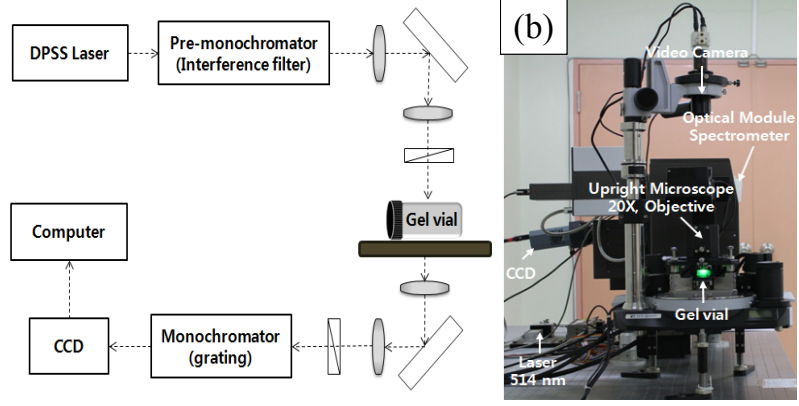

Figure 2. (a) Schematic diagram of a Raman spectrometer. (b) Analysis of polymethacylic acid gel using Nano Raman spectroscopy.

dose was located in the middle of the dosimeter. By combining 3 gel dosimeters into 1 group, examinations of radiation for absorption doses of $0,2,4,6,8,10,12,14,16,18$ and 20 Gy are conducted (Fig. 1). After the radiation examination, it was kept in cold storage for one night to fix polymerization in the gel.

SEM Image. The polymer gels with radiation of $0 \mathrm{~Gy}$ and 10 Gy were imaged with the Cryo FE-SEM (S-4700, Hitachi, Japan). A small quantity of collected gel was fixed at a carrier, and it was frozen in liquid nitrogen. The frozen gel was placed in a Cryogenic pre-processing system (MED $020 \mathrm{GBE}$, BalTec) for cutting with metal knife, and an etching process was carried out to remove remaining moisture for a clear image of the gel surface. After gel coating $(\mathrm{Pt})$, an image was acquired under the temperature $\left(-120^{\circ} \mathrm{C}\right)$ and vacuum $\left(1.6 \times 10^{-5}\right.$ Torr $)$ conditions.

Raman Spectrums. In order to understand the radiation characteristics of the gel, Raman data were acquisited using Nano Raman Spectroscopy (NTEGRA, NT-MDT, Russia). Fig. 2(a) indicates the schematic diagram of Nano Raman Spectrometer. A $532 \mathrm{~nm}$ Diode Pumped Solid State Laser (DPSS) was used as a light source, and a Charge-Coupled Device (CCD) was applied for a detector. Data acquisited from the Raman Spectrometer were analyzed by Origin ${ }^{\mathrm{TM}}$ (OriginLab Corp., MA, USA) and GRAMS software (Galactic Industries Corp., New Hampshire, USA). Each spectrum of Gelatin, MAA, HQ and THPC inserted to the gel was acquired to assign vibration bands of the polymer gel.

\section{Results and Discussion}

Gel Images Using Cryogenic SEM. After irradiation of 0 Gy and 10 Gy to the polymer gel dosimeter, images were taken
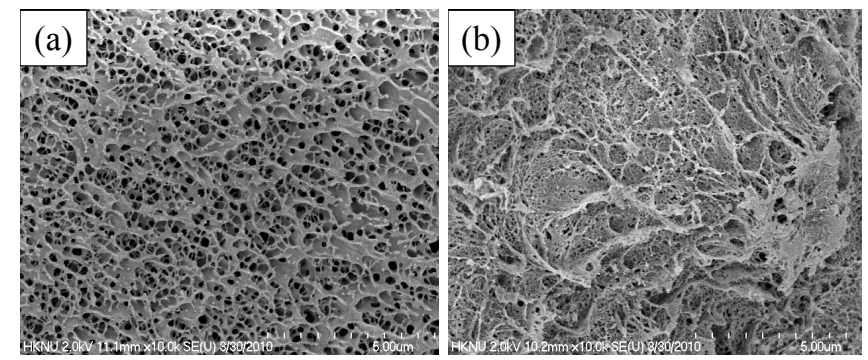

Figure 3. Cryo FE-SEM images of normoxic polymethacrylic acid gel dosimeter taken in a 5- $\mu \mathrm{m}$ scale for irradiation (a) 0 Gy and (b) 10 Gy.

by SEM in a $5-\mu \mathrm{M}$ scale. The polymer gel has a network structure $^{10}$ at first as shown in Fig. 3(a). As the dose was increased, it was shown that most network structures were disappeared as shown in Fig. 3(b). This is because MAA monomers turned to PMAA polymers while filling network holes during irradiation of the PMAA gel dosimeter.

Decrease of Methacrylic acid (MAA) and Increase of Polymethacrylic Acid (PMAA). When normoxic polymethacrylic acid gel is irradiated, the monomer methacrylic acid is transformed to a polymer polymethacrylic acid. The polymerization is initiated by the $\mathrm{OH}$ radical produced from the water molecule with the irradiation. And then, the polymerization occurs from MAA molecules by sequential radical reactions. The amount of dosage which derives full reaction was investigated by monitoring the polymerization process using Raman spectroscopy in this study. For better understanding on the behavior of the Raman bands resulted from the specific bonds in the process of polymerization, the spectra and the schematic diagram are shown in Fig. 4 and Fig. 5, respectively. The numerical vibrational frequencies are placed on the proper bands and their related bonds. Fig. 4 (a), (b), and (c) indicate the changes in intensity of vibrational modes for monomers, and (d) indicates, for the polymer during polymerization. Owing to the nonexistence of a cross-linker, precipitated polymer fills the network structure of the gelatin. In addition, all the molecules except MAA are independent of the dosage. Therefore, the bands appeared in Fig. 4 were normalized to that of the gelatin.

A band at $801 \mathrm{~cm}^{-1}$ in Fig. 4(a) is caused by symmetric stretching mode of $\mathrm{C}-\mathrm{COOH}$ which corresponds to a bond between carboxylic carbon and double bond carbon as shown in Fig. $5 .^{11}$ This mode is a polarized one, which is known to be very sensitive to the environmental change such as dissociation of carboxylic acid. ${ }^{12}$ The bond connecting two carbons is formed by the participation of a $\mathrm{SP}^{2}$ hybrid orbital, and the bond force constant becomes weaker as it changes to a $\mathrm{SP}^{3}$ hybrid orbital while polymerization proceeds. Thus, since this polymerization induces a decrease in numbers of monomer mode, the decrease in intensity for that vibrational mode is expected according to the increment of dose, which is experimentally shown in Fig. 4(a). A band clearly shown in 0 Gy became gradually decreased in intensity and finally insignificant at $18 \mathrm{~Gy}$. This result indicates that most monomers are transformed to polymers at 18 Gy dose.

A band at $1639 \mathrm{~cm}^{-1}$, which is another band for verifying transformation from monomer to polymer, is caused by a $\mathrm{C}=\mathrm{C}$ 
(a)

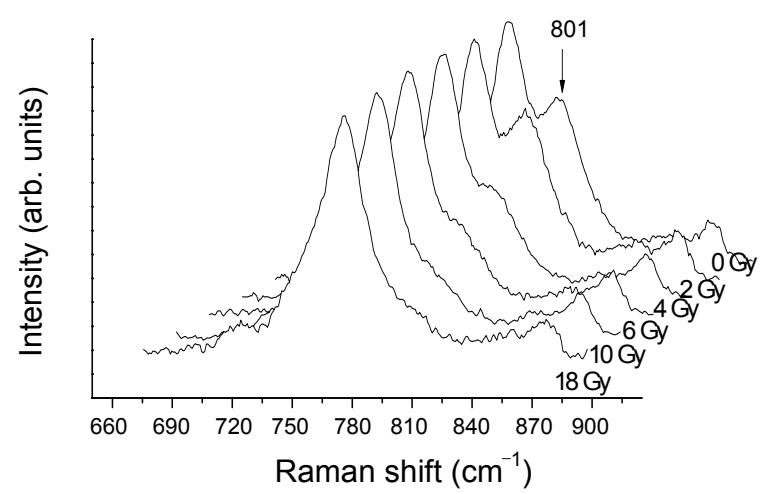

(c)

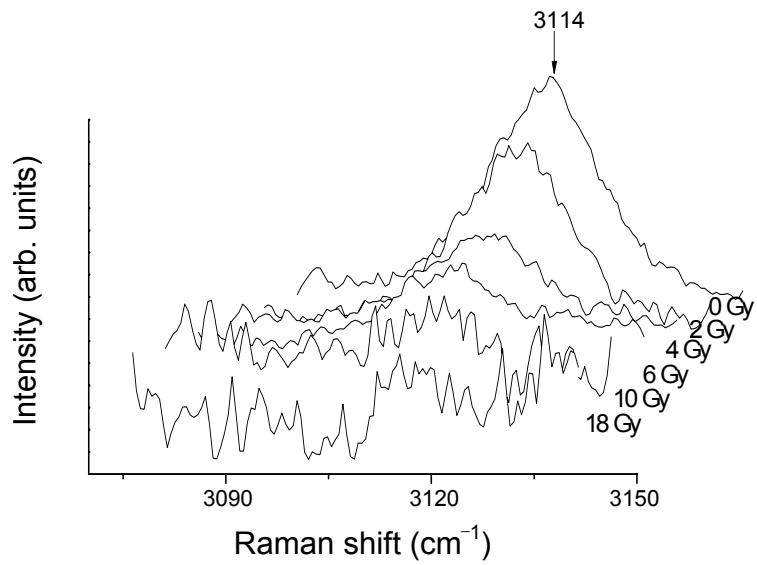

(b)

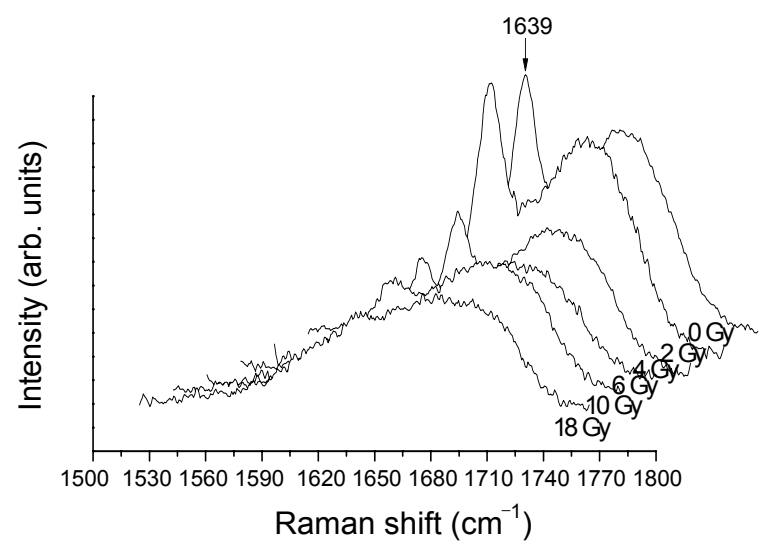

(d)

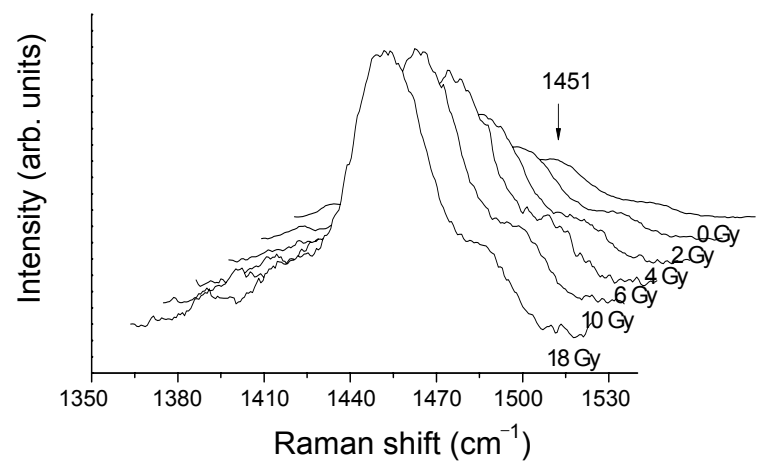

Figure 4. Polymer development observed in Nano Raman spectra of irradiated, normoxic polymethacrylic acid gel. (a) $\mathrm{C}-\mathrm{C}$ mode, (b) $\mathrm{C}=\mathrm{C}$ mode, (c) $\mathrm{CH}_{2}$ stretching mode of methacrylic acid and (d) $\mathrm{CH}_{2}$ bending mode of polymethacrylic acid.

stretching vibrational mode. ${ }^{11,13}$ As shown in Fig. 5, a $\mathrm{C}=\mathrm{C}$ double bond is changed to single bond when a monomer is transformed to a polymer. $\mathrm{A} \mathrm{C}=\mathrm{C}$ stretching vibrational mode of the original MAA appears at $1639 \mathrm{~cm}^{-1}$. However, as the polymerization proceeds, it gradually changes to a single bond, which also causes a weakening of the band intensity. Therefore, the intensity of this band gradually decreases, and finally disappears at $18 \mathrm{~Gy}$, similar to the band at $801 \mathrm{~cm}^{-1}$ in Fig. 4(b).

A band appearing at $3114 \mathrm{~cm}^{-1}$ is assigned to the asymmetric vinyl $\mathrm{CH}$ stretching mode of the MAA in Fig. 5. ${ }^{14}$ This band is also a vibrational mode which allows checking of the level of polymerization of the MAA. As polymerization carries on, this band disappears with the change from $\mathrm{SP}^{2}$ to $\mathrm{SP}^{3}$ hybrid orbital. Especially, the band for this vibrational mode does not overlap with other bands, therefore, it faithfully reflects the behavior of the MAA. Unlike the other bands, the drop in intensity of this band was completed at 10 Gy as shown in Fig. 4(c).

The band at $1451 \mathrm{~cm}^{-1}$ is attributed to the $\mathrm{CH}_{2}$ vibrational bending mode of PMAA. Thus, as MAA is transformed to PMAA, the intensity of this band is gradually increased.

Changes in the Raman intensity with respect to the absorbed dose are plotted in Fig. 6 to find out the sensitivity parameters for the decay of MAA and the growth of PMAA. Firstly, each band was normalized, and is fitted into the exponential decay function. It is known that a decrease in monomer in accordance with dosage generally follows a monoexponential decay curve. ${ }^{8}$<smiles>CC=CC(=O)O</smiles>

(a)

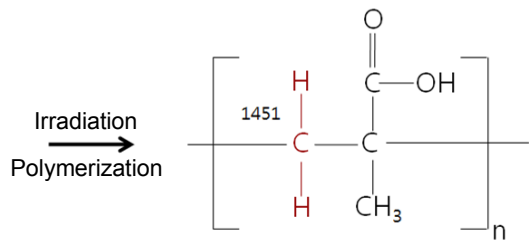

(b)
Figure 5. The chemical structure of (a) Methacrylic acid, (b) Polymethacrylic acid.

Therefore, the results of the experiment herein were fitted by the monoexponential fitting function provided by Origin ${ }^{\mathrm{TM}}$.

$$
\mathrm{y}=\mathrm{y}_{0}+\mathrm{a} e^{-\mathrm{D} / \mathrm{s}}
$$

Here, $\mathrm{y}$ indicates the number of monomers remaining after the absorption of a dose $D, \mathrm{y}_{0}$ is a value for background, $a$ for proportional constant and $s$ indicates sensitivity parameter.

As shown in Fig. 6(a), the band at $801 \mathrm{~cm}^{-1}$ seems to follow relatively the monoexponential decay curve, and the sensitivity parameter for it is $5.3 \pm 1.5 \mathrm{~Gy}$. It is clear that this value is much shorter than the 8.36 Gy and 12 Gy obtained by Baldock et al. ${ }^{8}$ and Jirasek et al., ${ }^{7}$ respectively, for AA., being in good agreement with the report that MAA has higher sensitivity than AA. This fact is supported by the results of other two vibrational 
(a)

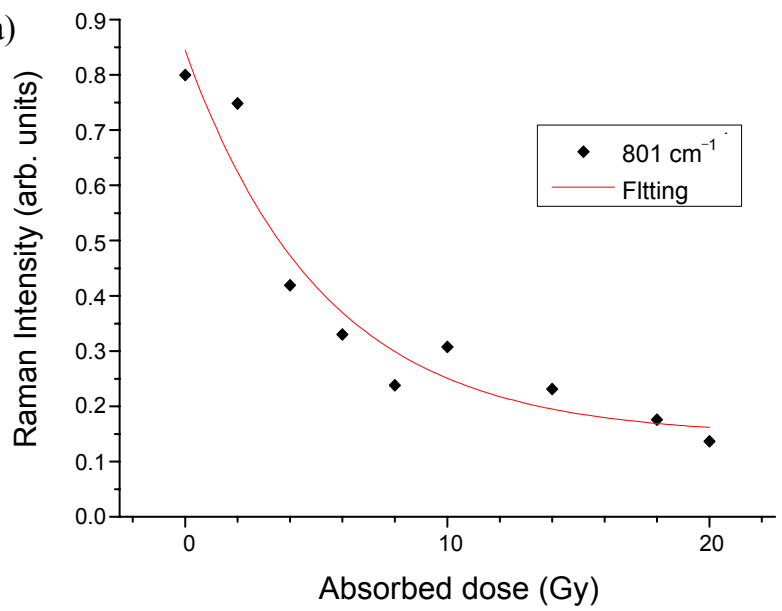

(c)

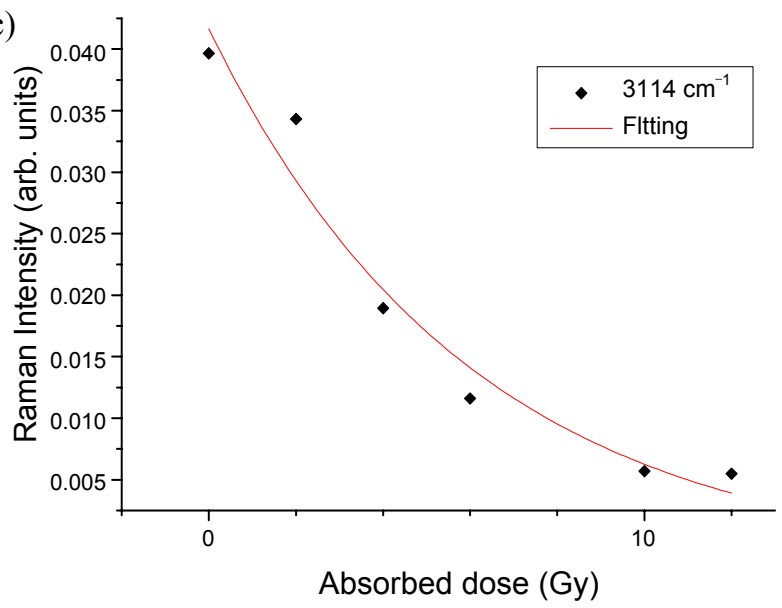

(b)

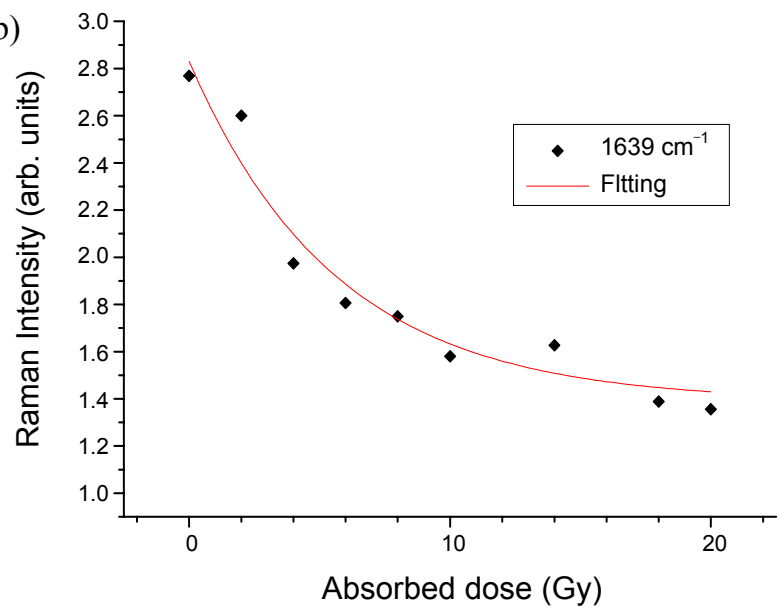

(d)

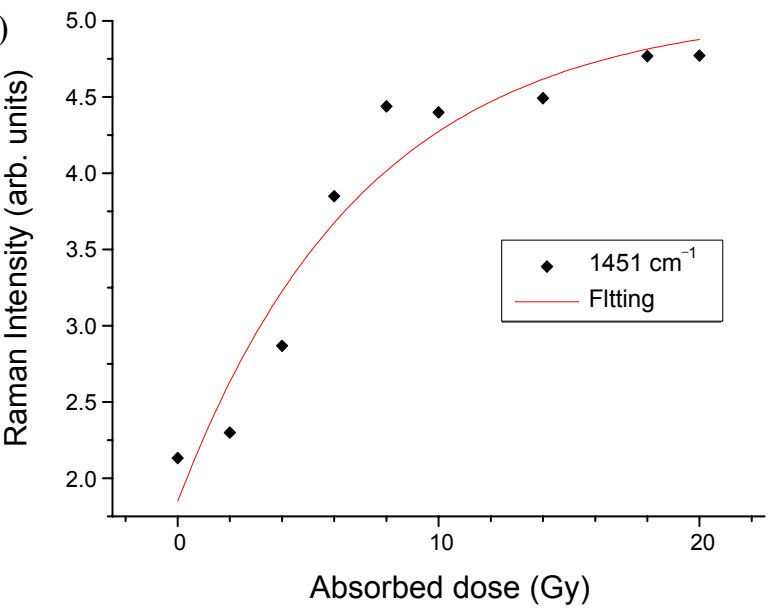

Figure 6. Trend of variation (a) $801 \mathrm{~cm}^{-1}$, (b) $1639 \mathrm{~cm}^{-1}$, (c) $3114 \mathrm{~cm}^{-1}$ and (d) $1451 \mathrm{~cm}^{-1}$.

modes of MAA at 1639 and $3114 \mathrm{~cm}^{-1}$.

The sensitivity parameters for the bands at 1639 and 3114 $\mathrm{cm}^{-1}$ are found to be $5.6 \pm 1.6 \mathrm{~Gy}$, and $6.0 \pm 2.6 \mathrm{~Gy}$, respectively, and their fitting curves are shown in Fig. 6(b) and Fig. 6(C). The value for the band at $1639 \mathrm{~cm}^{-1}$ is almost the same as that of the band at $801 \mathrm{~cm}^{-1}$ within the margins of error as expected. However, the value for the band at $3114 \mathrm{~cm}^{-1}$ shows somewhat higher uncertainty of \pm 2.6 . This is probably due to the narrow fitting range as well as the low $\mathrm{S} / \mathrm{N}$ ratio. As can be seen in Fig. 6 (C), the fitting was carried out upto 12 Gy instead of 20 Gy unlike the other bands. It is taken for granted that less data points result in hjgher uncertainty. This fact, however, does not change the conclusion that MAA has higher sensitivity than AA.

Unlike the decrease of monomers, increase of polymers is carried on along the equation below,

$$
\mathrm{y}=\mathrm{y}_{0}+\mathrm{a}\left(1-e^{-\mathrm{D} / \mathrm{s}}\right)
$$

The band at $1451 \mathrm{~cm}^{-1}$ represents the $\mathrm{CH}_{2}$ bending of polymethacrylic acid and gelatin, which has an $\mathrm{s}=7.2 \pm 2.3$ Gy shown in Fig. 6(d). Generally, as monomers decrease, the amount of polymers increases, so that the decreasing rate of monomer and the increasing rate of polymerization should be identical according to radiation level. ${ }^{7}$ However, if the change in gelatin follows according to radiation levels, it is difficult to identify that this value is caused by a pure polymer. Jirasek et. al. reported the result obtained using Raman spectroscopy that THPC reacted with the gelatin prior to radiation, causing a decrease in the $\mathrm{CH}_{2}$ bending mode of gelatin. ${ }^{15}$ Meanwhile, Lepage et. al. reported that changes in the gelatin did not appear until $50 \mathrm{~Gy}$ for THPC contained polyacrylamide gel. ${ }^{16}$ Upon summarizing the research, the changes in gelatin caused by THPC is not activated during irradiation, but completed before radiation, Therefore, the band at $1451 \mathrm{~cm}^{-1}$ is applicable for the pure $\mathrm{CH}_{2}$ bending mode of polymethacrylic acid, in which the $7.2 \pm 2.3$ Gy reflects the formation of a pure polymer, and it is seen that this result conforms with the decreasing rate of monomers within the range of error.

\section{Conclusions}

Radiation is applied to a nomoxic polymethacrylic acid gel based on a methacrylic acid as monomer. From comparison and analysis of the SEM images and Raman spectrum, the following conclusion is achieved.

A 3-dimensional network structure was shown in the 0 Gy SEM image of the gel dosimeter, and it was able to monitor 
changes in the structure from MAA to PMAA with being surrounded by the gelatin network structure when 10 Gy of radiation is irradiated.

From observation of features of the decreasing rate of monomer and the increasing rate of polymerization, three vibrational modes located in the monomer showed a decreasing trend, while the polymer showed an increasing trend. Intensity changes in the vibrational modes according to dosage followed a monoexponential curve shape, while sensitivity parameters for monomer bands and polymer band were ranged in $6.0 \pm 2.6 \mathrm{~Gy}$ and 7.2 $\pm 2.3 \mathrm{~Gy}$, respectively, showing a relatively good conformity of the decreasing rate of monomer and the increasing rate of polymerization within the range of error.

Raman spectroscopy was applied for the first time to the methacrylic acid based gel dosimeter, and it showed that the methacrylic acid based gel dosimeter is more sensitive than acrylamid acid based gel, being in good agreement with the results of NMR spectroscopy. Since it is possible to achieve the information at the molecular level from the application of vibration spectroscopy, i.e. Raman spectroscopy, for observation of chemicophysical changes in the gel, it is expected to contribute to the development of a 3-dimensional radiation dosimeter which is efficient for radiation treatment.

Acknowledgments. This work was supported by National Research Foundation of Korea Grant funded by the Korean Government (2009-0093757)

\section{References}

1. Chung, S. Y.; Kim, Y. B.; Kwon, Y. H.; Lee, S. J. Korean Soc. Radiotherapeutic Technol. 2007, 19, 7.
2. Kim, B.; McAuley, J. Phys. 2006, 56, 35

3. Kang, H. J.; Cho, S. J.; Jeong, E.-K.; Kang, S. H.; Oh, Y. T.; Chun, M.; Kwon, S.-I. Korean J. Med. Phys. 1998, 9, 207.

4. De Deene, Y.; Vergote, K.; Claeys, C.; De Wagter, C. Phys. Med. Biol. 2006, 51, 653.

5. De Deene, Y.; Hanselaer, P.; De Wagter, C.; Achten, E.; De Neve, W. Phys. Med. Biol. 2000, 45, 859.

6. Colthup, N. B.; Daly, L. H.; Wiberley, S. E. Introduction to Infrared and Raman Spectroscopy; Academic Press: London, 1990.

7. Jirasek, A. I.; Duzenli, C.; Audet, C.; Eldridge, J. Phys. Med. Biol. 2001, 46, 151.

8. Baldock, C.; Rintoul, L.; Keevi, S. F.; Pope, J. M.; George, G. A. Phys. Med. Biol. 1998, 43, 3617.

9. Jirasek, A. I.; Duzenli, C. Phys. Med. Biol. 2001, 46, 1949.

10. Vlierberghe, S. V.; Cnudde, V.; Dubruel, P.; Masschaele, B.; Cosijns, A.; De Paepe, I.; Patric, J. S.; Jacobs, Hoorebeke, L. V.; Remon, J. P.; Schacht, E. Biomacromolecules 2007, 8, 331.

11. Halamus, T.; Wojciechowski, P. Polym. Adv. Technol. 2007, 18, 411.

12. Koenig, J. L.; Angood, A. C.; Semen, J.; Lando, J. B. J. Am. Chem. Soc. 1969, 91, 7250.

13. Gupta, M. K.; Bansil R, J. Polym. Sci., Polym. Lett. Ed. 1983, 21, 969.

14. Gupta, M. K.; Bansil, R. J. Polym. Sci., Polym. Phys. Ed. 1981, 19, 353.

15. Jirasek, A.; Hilts, M.; Shaw, C.; Baxter, P. Phys. Med. Biol. 2006, $51,1891$.

16. Lepage, M.; Whittaker, A. K.; Rintoul, L.; Baldock, C. Appl. Polym. Sci. 2001, 79, 1572.

17. Hilts, M.; Audet, M.; Duzenli, C.; Jirasek, A. Phys. Med. Biol. 2000, 45, 2559.

18. Hayashi, S.-I.; Yoshioka, M.; Usui, S.; Haneda, K.; Kondo, T.; McAuley, K. B.; Tominaga, T. Radiation Physics and Chemistry 2010, 79,803

19. Hurleya, C.; Venninga, A.; Baldock, C. Appl. Radiation and Isotopes 2005, 63, 443. 